\title{
O estado nutricional modera a associação entre apoio social, autoeficácia e tempo de atividade física dos adolescentes?
}

\author{
Does nutritional status moderate the association between social \\ support, self-efficacy, and physical activity time of adolescents?
}

Tayse Guedes Cabral (https://orcid.org/0000-0002-6731-3955) ${ }^{1}$

Ially Rayssa Dias Moura (https://orcid.org/0000-0001-8210-2059) ${ }^{1}$

José Cazuza de Farias Júnior (https://orcid.org/0000-0002-1082-6098) ${ }^{1}$

${ }^{1}$ Faculdade de Educação Física, Universidade Federal da Paraíba. Jardim Universitário $\mathrm{s} / \mathrm{n}$, Castelo Branco. 58051-900 João Pessoa PB Brasil. tayse_cabral@hotmail.com
Abstract The scope of this paper is to analyze the association between social support, self-efficacy and practice time of physical activity of different intensity (light, moderate, vigorous) and evaluate if these associations are moderated by the nutritional status of adolescents. It involved a cross-sectional study with 650 adolescents (10-14 years old, 52.9\% female) from João Pessoa, State of Paraíba. Social support was measured by a scale with five items for each source of support ( $\mathrm{fa}$ ther, mother and friends) and self-efficacy, with a scale of four items. The physical activity time was measured by means of an accelerometer and the nutritional status was assessed by body mass index. The results of the multiple linear regression analysis showed that social support of friends was positively and significantly associated with moderate intensity physical activity time ( $\beta=2.67$; CI95\%: 0.28; 5.07). The nutritional status did not moderate the association of social support and self-efficacy with the duration of physical activity $(p>0.05)$. The conclusion drawn is that adolescents who perceived that they are receiving more social support from friends tend to practice more moderate intensity physical activity, irrespective of their nutritional status.

Key words Motor activity, Social support, Self-efficacy, Adolescents
Resumo O objetivo foi analisar a associação entre o apoio social, autoeficácia e o tempo de prática de atividade física de diferentes intensidades (leve, moderada, vigorosa) e avaliar se essas associações são moderadas pelo estado nutricional dos adolescentes. Estudo transversal, com 650 adolescentes (10-14 anos de idade, 52,9\% do sexo feminino) de João Pessoa-PB. O apoio social foi mensurado por uma escala com cinco itens para cada fonte de apoio (pai, mãe e amigos) e a autoeficácia com escala de quatro itens. O tempo de atividade física foi mensurado por meio de acelerômetro e o estado nutricional determinado a partir do indice de massa corporal. Na análise de regressão linear múltipla o apoio social dos amigos se associou de forma positiva e significativa com o tempo de prática de atividade física de intensidade moderada ( $\beta=2,67$; IC95\%: 0,28; 5,07). O estado nutricional não moderou a associação do apoio social $e$ da autoeficácia com o tempo de atividade física ( $p>0,05)$. Adolescentes que percebiam receber mais apoio social dos amigos apresentaram maior prática de atividade física de intensidade moderada, independentemente do seu estado nutricional. Palavras-chave Atividade motora, Apoio social, Autoeficácia, Adolescentes 


\section{Introdução}

A quantidade de estudos para identificar os fatores correlatos e/ou determinantes da atividade física em adolescentes tem crescido de forma expressiva nas últimas duas décadas ${ }^{1,2}$. Entretanto, recomenda-se, além de identificar esses fatores/determinantes, analisar se as suas relações com a prática de atividade física variam com as características dos adolescentes (identificar moderadores) ${ }^{1}$.

$\mathrm{O}$ apoio social e a autoeficácia para a prática de atividade física estão entre os fatores associados a maiores níveis de atividade física ${ }^{1,2}$; essas associações têm sido moderadas pelo sexo e pela idade dos adolescentes ${ }^{3}$, mas uma possível moderação pelo estado nutricional foi pouco investigada, e os resultados dos estudos foram controversos $^{4-8}$.

O apoio social para a prática de atividade física é uma forma de assistência que auxilia na manutenção e/ou adoção desse comportamento, podendo ser fornecido por diversas fontes (por exemplo, pais, amigos) e de diferentes formas (emocional, instrucional e instrumental) ${ }^{9}$. A autoeficácia refere-se à crença que a pessoa tem quanto a sua capacidade para adotar e/ou manter determinada prática de atividade física diante da presença de barreiras/dificuldades ${ }^{10}$.

A prevalência de excesso de peso corporal em adolescentes é elevada: cerca de $50 \%$ em países de renda alta e $21 \%$ em países de renda baixamédia $^{11}$. Dados da Pesquisa Nacional de Saúde Escolar (PeNSE, 2015) indicaram que 23,7\% dos adolescentes brasileiros têm excesso de peso, sendo $26,9 \%$ na região Nordeste ${ }^{12}$. Em geral, adolescentes com excesso de peso subestimam as suas habilidades para a prática de atividade física e esportes $^{13}$, têm mais interações sociais negativas $^{14}$, são menos ativos fisicamente ${ }^{15}$, pertencem a famílias de condições socioeconômicas mais baixas $^{12}$, percebem mais barreiras e menos apoio social para a prática de atividade física ${ }^{8}$. Sendo assim, é possível que as associações entre apoio social, autoeficácia e prática de atividade física variem de acordo com o estado nutricional.

Os resultados dos estudos sobre o papel moderador do estado nutricional na associação do apoio social e da autoeficácia com a prática de atividade física em adolescentes ainda são inconsistentes ${ }^{4-8}$. Os estudos identificados foram realizados com adolescentes de faixa etária restrita ${ }^{(4,7)}$, do sexo feminino ${ }^{4,8}$, mensuraram a atividade física com questionário ${ }^{6,8}$, e os que utilizaram acelerômetros não analisaram as atividades de intensidade leve $e^{4,5,7}$. Desse modo, este estudo analisou as associações entre o apoio social, a autoeficácia e o tempo de prática de atividade física em diferentes intensidades (leve, moderada, vigorosa); e avaliou se elas eram moderadas pelo estado nutricional dos adolescentes.

\section{Métodos}

Estudo transversal que analisou dados da linha de base do Estudo Longitudinal sobre Comportamento Sedentário, Atividade Física, Alimentação e Saúde dos Adolescentes Estudo (LONCAAFS), que teve como população-alvo os adolescentes de ambos os sexos, matriculados no $6^{\circ}$ ano do ensino fundamental de escolas públicas de João Pessoa, em 2014. Informações detalhadas sobre o LONCAAFS estão em outra publicação ${ }^{16}$. Esta pesquisa foi aprovada pelo Comitê de Ética em Pesquisa com Seres Humanos da Universidade Federal da Paraíba.

Para o cálculo de tamanho da amostra do LONCAAFS, foram adotados os seguintes parâmetros: população de referência igual a 9.520 escolares no $6^{\circ}$ ano do ensino fundamental de escolas públicas da cidade de João Pessoa-PB; prevalência do desfecho igual a $50 \%$ - projeto com múltiplos desfechos, como no caso do LONCAAFS, esse procedimento permite alcançar uma amostra de maior tamanho para um mesmo de erro amostral máximo aceitável; intervalo de confiança de 95\%; erro máximo aceitável de quatro pontos percentuais; efeito de desenho (deff) igual a dois ${ }^{17}$. Com base nesses parâmetros, o tamanho mínimo da amostra foi de 1.130 adolescentes. Foram acrescidos $40 \%$ para compensar possíveis perdas e recusas durante os quatro anos de coleta de dados, resultando em uma amostra de 1.582 adolescentes.

No presente estudo, foram utilizados dados de uma subamostra de adolescentes que utilizou acelerômetro, representando aproximadamente $70 \%$ dos casos da amostra. Para tanto, selecionaram-se aleatoriamente 17 das 28 escolas da amostra, distribuídas proporcionalmente por região geográfica (norte, sul, leste e oeste) e número de escolares no $6^{\circ}$ do ensino fundamental, em cada escola. A utilização de acelerômetro em uma subamostra foi decorrente do número de equipamentos disponíveis, de membros na equipe de coleta de dados e do tempo disponível para tal. Entretanto, essa subamostra manteve a mesma distribuição proporcional dos escolares observadas na população e amostra, e não apresentou diferenças significativas nas variáveis estudadas 
em comparação aos dados da amostra ${ }^{16}$. Informações detalhadas sobre a amostragem estão apresentadas na Figura 1.

A coleta de dados foi realizada de fevereiro a junho e de agosto a dezembro de 2014, na escola, por equipe treinada; consistiu na aplicação de questionário, por entrevista "face a face", realiza- ção de medidas antropométricas e uso de acelerômetros.

As variáveis sociodemográficas analisadas foram sexo (masculino e feminino); idade centesimal (diferença entre a data de nascimento e a data de coleta de dados, agrupada em 10-11 e 1214 anos); classe econômica (critérios da $\mathrm{ABEP}^{18}$,

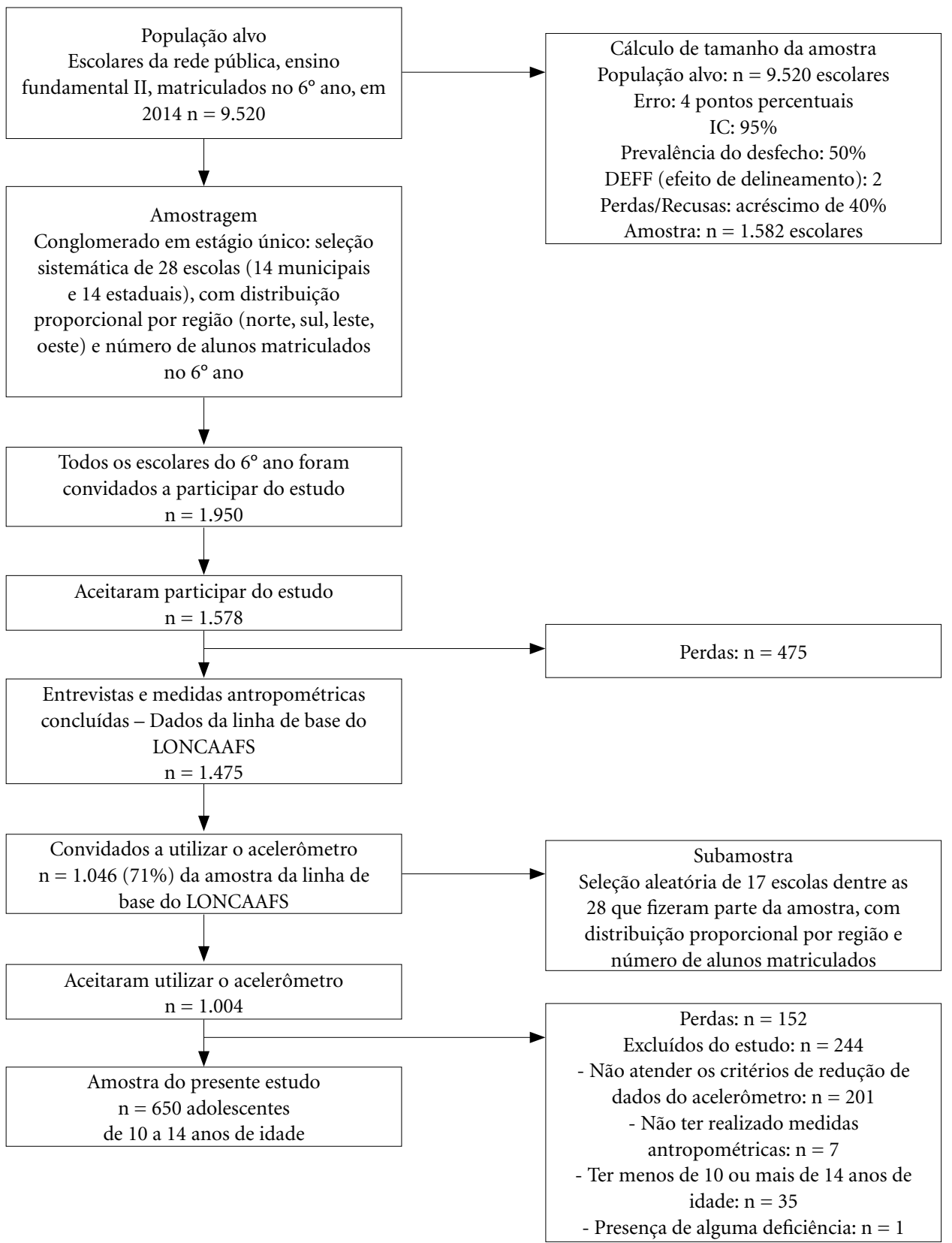

Figura 1. Flowchart do procedimento de amostragem. 
que agrupa as pessoas nas classes "A" a "E", posteriormente reagrupadas em "A/B" [classe alta] e "C/D/E” [média-baixa]); e escolaridade da mãe (fundamental incompleto, fundamental completo, médio completo ou superior).

$\mathrm{O}$ apoio social foi mensurado por meio de uma escala ${ }^{19}$ de cinco itens para cada fonte de apoio (pai, mãe e amigos). Os adolescentes informaram a frequência (nunca $=1$, raramente $=2$, frequentemente $=3$ ou sempre $=4$ ) com que o pai, a mãe e os amigos forneciam algum tipo de apoio social durante uma semana típica, por exemplo: "Durante uma semana normal com que frequência seu [pai/mãe/amigos] ... [incentiva, pratica junto, transporta/convida, assiste e comenta] você a praticar atividade física?". Foram produzidos escores de apoio social para cada fonte de apoio, baseados no somatório das respostas dos itens das respectivas escalas, os quais variaram de 5 (menor nível) a 20 pontos (maior nível de apoio social).

A autoeficácia foi mensurada por escala ${ }^{19}$ de quatro itens, e os adolescentes referiam o quanto se percebiam capazes de praticar atividade física na maioria dos dias da semana na presença de barreiras ( $\operatorname{sim}=2$ e não=1), por exemplo: "Você conseguiria praticar atividade física ou esportes na maioria dos dias da semana, mesmo que não tivesse ninguém para ir com você?”. Foram produzidos escores de autoeficácia com base no somatório da resposta de cada item, os quais variaram de 4 (menor nível) a 8 (maior nível de autoeficácia).

As escalas de apoio social e de autoeficácia apresentaram níveis satisfatórios de fidedignidade (apoio social - Alpha de Cronbach: pai=0,75; mãe $=0,80$; amigo $=0,77$; coeficiente de correlação intraclasse: pai $=0,80 ;$ mãe $=0,78$; amigo $=0,75$; autoeficácia - Índice de fidedignidade combinada=0,38; Kappa Pabak=0,68) e de validade de construto (análise fatorial confirmatória: apoio social - cargas fatoriais $>0,59$; autoeficácia - cargas fatoriais de 0,33 a 0,38$)^{19}$.

A medida da massa corporal foi realizada em balança digital, e a da estatura, em estadiômetro. Foram realizadas três medidas, e o valor médio foi adotado como resultado para fins de análise. A partir do índice de massa corporal (IMC - massa corporal $[\mathrm{kg}] /$ estatura $[\mathrm{m}]^{2}$ ), determinado com base nas medidas de massa e estatura, os adolescentes foram classificados como "sem excesso de peso" (baixo peso + peso normal) e "com excesso de peso" (sobrepeso + obesidade), conforme critérios da Organização Mundial da Saúde ${ }^{20}$.

A atividade física e o comportamento sedentário foram mensurados por meio de acelerô- metros da Actigraph GT3X+. O adolescente foi orientado a utilizá-lo por sete dias consecutivos, fixado no lado direito da cintura por cinto elástico, retirando-o para dormir, tomar banho, realizar atividades aquáticas e lutas com quedas (devido ao risco de lesão). Na redução dos dados, foi utilizado o programa ActiLife 6.12, conforme os critérios que seguem: usar por pelo menos oito horas por dia, três ou mais dias da semana, sendo pelo menos um dia de final de semana ${ }^{16}$, epoch de 15 segundos e período de não uso de $60 \mathrm{minu}$ tos ou mais com zeros consecutivos. Os pontos de corte de Evenson et al. ${ }^{21}$ foram utilizados para definir o tempo em comportamento sedentário: $\leq 100$ counts/minuto; atividade física leve: 1012.295 counts/minuto; moderada: 2.296-4.011 counts/minuto; vigorosa: $\geq 4.012$ counts/minuto; moderada a vigorosa: $\geq 2.296$ counts/minuto.

$\mathrm{Na}$ determinação do tempo de comportamento sedentário e de atividade física de diferentes intensidades (leve, modera e vigorosa), em horas por dia, calculou-se a média ponderada: tempo médio (hora/dia) em dias de semana (segunda a sexta-feira) multiplicado por 5 , e por 2 para os dias de final de semana (sábado e/ou domingo), dividindo-se o somatório por 7 (obter o tempo em minutos/dia).

Os critérios de exclusão adotados foram: estar fora da faixa etária de interesse do estudo ( $<10$ e $>14$ anos de idade); ter alguma deficiência que impedisse ou limitasse a prática de atividade física ou resposta ao questionário; não atender aos critérios de redução dos dados do acelerômetro; não ter realizado as medidas de estatura e/ou massa corporal e; não ter respondido as questões sobre apoio social e autoeficácia.

$\mathrm{Na}$ análise descritiva dos dados, foi utilizada a distribuição por frequência para as variáveis qualitativas, e a média e o desvio padrão para as quantitativas. A regressão linear bruta e ajustada foi utilizada para associar o apoio social do pai, da mãe e dos amigos e a percepção de autoeficácia (variáveis independentes) ao tempo de atividade física leve, moderada, vigorosa e moderada a vigorosa (variáveis dependentes). O método utilizado na seleção das variáveis para o modelo ajustada foi o Forward, e os potenciais fatores de confusão utilizados foram sexo, idade, classe econômica, índice de massa corporal (quando não estava sendo tratado como moderador) e comportamento sedentário.

Para testar a presença de interação entre o estado nutricional (IMC), apoio social e autoeficácia, foi criado um termo de interação multiplicando o escore de cada fonte de apoio e de 
autoeficácia pelo estado nutricional do adolescente, por exemplo: apoio social do pai ${ }^{\star}$ estado nutricional (com excesso de peso corporal $=1$ e sem excesso de peso corporal $=0$ ). Valores de $\mathrm{p}<0,05$ para o coeficiente de regressão do termo de interação indicaram a presença de interação. No caso de interação presente, as análises seriam estratificadas pelo estado nutricional, permitindo avaliar a magnitude e o sentido da associação do apoio social e da autoeficácia com a atividade física entre esses dois grupos. As análises foram realizadas no Stata 13 . O nível de significância foi de $\mathrm{p}<0,05$; o intervalo de confiança, de $95 \%$.

\section{Resultados}

Para o presente estudo, foram selecionados 1.046 adolescentes, sendo que 152 foram considerados como perdas e 244 foram excluídos, resultando em uma amostra final de 650 adolescentes. A proporção de adolescentes excluídos das análises por não atenderem aos critérios de redução dos dados do acelerômetro foi maior nos que tinham de 12 a 14 anos de idade $(38,7 \%)$ comparativamente aos de 10 a 11 anos $(19,3 \%, p=0,02)$. Porém, não foram identificadas diferenças significativas $(\mathrm{p} \geq 0,05)$ para o apoio social, a autoeficácia e o tempo de prática de atividade física (dados não apresentados em tabelas).

A amostra do presente estudo foi composta por adolescentes de 10 a 14 anos de idade, de ambos os sexos. A maioria era do sexo feminino $(52,9 \%)$ e pertencia à classe econômica média-baixa $(63,6 \%)$, cerca de um terço era filho de mães que concluíram pelo menos o ensino médio. Verificou-se que $35,5 \%$ tinham excesso de peso corporal, tempo médio de atividade física moderada-vigorosa de 202,5 minutos por semana (desvio padrão=137,9) e 7 horas por dia (desvio padrão $=2,1)$ de tempo em comportamentos sedentários (Tabela 1). O tempo médio de uso do acelerômetro por dia foi de 12 horas (desvio padrão $=3,0)$ (dados não apresentados em tabelas).

$\mathrm{Na}$ análise bruta, o apoio social dos amigos (atividade física moderada: $\beta=3,79$; IC95\%: 1,$77 ; 5,80$ e atividade física moderada-vigorosa: $\beta=4,32$; IC95\%: 1,$72 ; 6,92)$ e a autoeficácia (atividade física moderada: $\beta=10,80$; IC95\%: 3,$33 ; 18,29$ e atividade física moderada-vigorosa: $\beta=11,92$; IC95\%: 2,$28 ; 21,56)$ associaram-se de forma positiva e significativa com o tempo de prática de atividade física (Tabela 2 ).

$\mathrm{Na}$ análise ajustada, apenas o apoio social dos amigos se manteve associado ao tempo de práti- ca de atividade física moderada ( $\beta=2,67$; IC95\%: $0,28 ; 5,07)$. O estado nutricional não apresentou interação significativa $(\mathrm{p} \geq 0,05)$ com $\mathrm{o}$ apoio social e a autoeficácia na associação com o tempo de prática de atividade física de diferentes intensidades (Tabela 2). Cálculos realizados a posterio$r i$ indicaram que a amostra deste estudo $(\mathrm{n}=650$ adolescentes) apresentou poder estatístico igual a 1,0 (1-Beta), para todos os modelos de regressão (considerando valores dos coeficientes de regressão - $\mathrm{R}^{2}$, variando de 0,14 a 0,25 , com 14 variáveis preditoras em cada um, e nível de significância de $5 \%)$.

\section{Discussão}

Adolescentes que recebiam mais apoio social dos amigos apresentaram maior tempo de prática de atividade física de intensidade moderada. $\mathrm{O}$ apoio social dos pais e a percepção de autoeficácia não se associaram ao tempo de prática de atividade física para todas as intensidades. A hipótese de que o estado nutricional dos adolescentes seria um moderador da associação entre apoio social, autoeficácia e prática de atividade física não foi confirmada no presente estudo.

$\mathrm{O}$ apoio social fornecido pelos pais e os amigos e a percepção de autoeficácia têm sido consistentemente associados a maiores níveis de prática de atividade física em adolescentes ${ }^{1,2}$. Aumentos nos níveis de apoio social e de autoeficácia, em resposta às ações desenvolvidas em intervenções, têm sido acompanhados de aumentos na prática de atividade física dos adolescentes ${ }^{1}$. Esses achados reforçam a importância desses dois construtos para a prática de atividade física em adolescentes.

No presente estudo, apenas o apoio social dos amigos se associou a um maior tempo de prática de atividade física de intensidade moderada dos adolescentes. Esse resultado pode estar relacionado ao fato de que, com o passar da idade, os adolescentes passam a ter maior convívio e praticar suas atividades físicas em companhia dos amigos?. O apoio social dos amigos pode aumentar em até três vezes as chances de os adolescentes serem fisicamente mais ativos ${ }^{22}$. Desse modo, o apoio social desse grupo social parece exercer maior influência na atividade física dos adolescentes comparativamente ao dos pais, sobretudo com o passar da idade na adolescência, ${ }^{9,22}$.

Os amigos podem influenciar a prática de atividade física dos seus pares adolescentes por diferentes formas/meios: norma social - os ado- 
Tabela 1. Características sociodemográficas, estado nutricional, comportamento sedentário, atividade física, apoio social do pai, mãe, dos amigos e autoeficácia de adolescentes de João Pessoa, Paraíba, 2014.

\begin{tabular}{|c|c|c|c|c|}
\hline Variáveis & \multicolumn{2}{|c|}{$\mathbf{n}$} & \multicolumn{2}{|c|}{$\%$} \\
\hline \multicolumn{5}{|l|}{ Sexo } \\
\hline Masculino & \multicolumn{2}{|c|}{306} & \multicolumn{2}{|c|}{47,1} \\
\hline Feminino & \multicolumn{2}{|c|}{344} & \multicolumn{2}{|c|}{52,9} \\
\hline \multicolumn{5}{|l|}{ Faixa etária } \\
\hline 10 a 11 anos & \multicolumn{2}{|c|}{413} & \multicolumn{2}{|c|}{63,3} \\
\hline 12 a 14 anos & \multicolumn{2}{|c|}{237} & \multicolumn{2}{|c|}{36,7} \\
\hline \multicolumn{5}{|l|}{ Classe econômica* } \\
\hline $\mathrm{A} / \mathrm{B}$ & \multicolumn{2}{|c|}{209} & \multicolumn{2}{|c|}{36,4} \\
\hline $\mathrm{C} / \mathrm{D} / \mathrm{E}$ & \multicolumn{2}{|c|}{365} & \multicolumn{2}{|c|}{63,6} \\
\hline \multicolumn{5}{|l|}{ Escolaridade da mãe* } \\
\hline Fundamental incompleto & \multicolumn{2}{|c|}{190} & \multicolumn{2}{|c|}{34,9} \\
\hline Fundamental completo & \multicolumn{2}{|c|}{163} & \multicolumn{2}{|c|}{30,0} \\
\hline Médio completo ou superior & \multicolumn{2}{|c|}{191} & \multicolumn{2}{|c|}{35,1} \\
\hline \multicolumn{5}{|l|}{ Estado nutricional } \\
\hline Com excesso de peso corporal & \multicolumn{2}{|c|}{231} & \multicolumn{2}{|c|}{35,5} \\
\hline \multirow[t]{2}{*}{ Sem excesso de peso corporal } & \multicolumn{2}{|c|}{419} & \multicolumn{2}{|c|}{64,5} \\
\hline & Média & $\mathrm{dp}$ & Mínimo & Máximo \\
\hline Comportamento sedentário (horas/dia) & 7,0 & 2,1 & 1,6 & 17,4 \\
\hline \multicolumn{5}{|l|}{ Atividade física (minutos/semana) } \\
\hline Leve & $2.153,7$ & 621,3 & 501 & 3.764 \\
\hline Moderada & 170,3 & 107,3 & 3 & 672 \\
\hline Vigorosa & 32,3 & 42,0 & 1 & 318 \\
\hline Moderada a vigorosa & 202,5 & 137,9 & 7 & 838 \\
\hline \multicolumn{5}{|l|}{ Escore de apoio social (pontos) } \\
\hline Pai & 11,1 & 4,1 & 5 & 20 \\
\hline Mãe & 11,1 & 3,8 & 5 & 20 \\
\hline Amigos & 13,4 & 4,1 & 5 & 20 \\
\hline Escore de autoeficácia (pontos) & 6,2 & 1,1 & 4 & 8 \\
\hline
\end{tabular}

$\mathrm{dp}=$ desvio padrão. ${ }^{\star} \mathrm{O}$ somatório não corresponde ao total de casos da amostra devido a valores inexistentes (missing).

Fonte: Elaborada pelos autores.

lescentes praticam determinadas atividades físicas para poder fazer parte ou pertencer ao grupo social dos seus amigos; apoio social - são estabelecidas ou fortalecidas as relações de amizade entre os que compartilham as práticas de atividade física ${ }^{23}$; modelagem do comportamento - sentem-se atraídos para iniciar uma prática de atividade física porque os amigos estão envolvidos. A prática de atividade física dos amigos passa a atuar como modelo de comportamento entre os adolescentes ${ }^{24}$.

A ausência de associação entre o apoio social dos pais (pai e mãe) e o tempo de prática de atividade física pode ter ocorrido devido ao grupo estudado pertencer, predominantemente, a famílias de baixo nível socioeconômico. Pais com menor nível de escolaridade, fenômeno frequentemen- te identificado nesses estratos socioeconômicos, podem ter menor preocupação e atenção com a prática de atividade física de seus filhos devido à elevada carga de trabalho semanal e/ou ao menor nível de conhecimento sobre a importância de tal prática para a saúde ${ }^{25}$; e menor condição econômica para disponibilizar equipamentos, materiais e oportunidades de prática para os seus filhos ${ }^{26}$. Destaca-se, ainda, o fato de que pais mais pobres são fisicamente menos ativos, e o nível de atividade física deles está associado ao dos seus filhos ${ }^{1,26}$.

O nível socioeconômico está associado às condições de infraestrutura do bairro em que os adolescentes residem. Famílias de condições socioeconômicas mais baixas residem em bairros com menor infraestrutura para a prática de ati- 
Tabela 2. Regressão linear bruta e ajustada para associação entre tempo de prática de atividade física e apoio social do pai, mãe e amigos, e autoeficácia em adolescentes de João Pessoa, Paraíba, 2014.

\begin{tabular}{|c|c|c|c|c|c|c|c|c|}
\hline \multirow{2}{*}{ Análise bruta } & \multicolumn{2}{|r|}{ AFL } & \multicolumn{2}{|r|}{ AFM } & \multicolumn{2}{|r|}{ AFV } & \multicolumn{2}{|r|}{ AFMV } \\
\hline & $\boldsymbol{\beta}$ & IC95\% & $\boldsymbol{\beta}$ & IC95\% & $\boldsymbol{\beta}$ & IC95\% & $\boldsymbol{\beta}$ & IC95\% \\
\hline Apoio social do pai & 2,93 & $-9,95 ; 15,82$ & 1,81 & $-0,36 ; 3,99$ & 0,07 & $-0,75 ; 0,90$ & 1,89 & $-0,90 ; 4,68$ \\
\hline Apoio social da mãe & 0,75 & $-12,18 ; 13,67$ & 0,62 & $-1,58 ; 2,83$ & $-0,01$ & $-0,93 ; 0,76$ & 0,54 & $-2,30 ; 3,37$ \\
\hline Apoio social do amigo & 1,03 & $-10,72 ; 12,78$ & 3,79 & 1,$77 ; 5,80$ & 0,53 & $-0,26 ; 1,33$ & 4,32 & 1,$72 ; 6,92$ \\
\hline Autoeficácia & 2,40 & $-41,22 ; 46,03$ & 10,80 & 3,$33 ; 18,29$ & 1,11 & $-1,84 ; 4,06$ & 11,92 & 2,$28 ; 21,56$ \\
\hline Análise ajustada & $\boldsymbol{\beta}$ & IC95\% & $\beta$ & IC95\% & $\boldsymbol{\beta}$ & IC95\% & $\boldsymbol{\beta}$ & IC95\% \\
\hline Apoio social do pai ${ }^{\star}$ & $-2,60$ & $-19,32 ; 14,11$ & $-0,40$ & $-3,12 ; 2,32$ & $-0,27$ & $-1,34 ; 0,81$ & $-0,66$ & $-4,10 ; 2,77$ \\
\hline Apoio social da mãe & 1,06 & $-16,50 ; 18,62$ & 0,10 & $-2,75 ; 2,96$ & $-0,10$ & $-1,22 ; 1,03$ & 0,01 & $-3,60 ; 3,62$ \\
\hline Apoio social do amigo ${ }^{\star * \star}$ & 8,12 & $-6,58 ; 22,81$ & 2,67 & 0,$28 ; 5,07$ & 0,13 & $-0,81 ; 1,07$ & 2,81 & $-0,22 ; 5,83$ \\
\hline Autoeficácia ${ }^{* * * *}$ & $-31,95$ & $-82,58 ; 18,69$ & 6,55 & $-1,69 ; 14,79$ & $-0,38$ & $-3,62 ; 2,86$ & 6,17 & $-4,24 ; 16,58$ \\
\hline Análise de interação & $\boldsymbol{\beta}$ & IC95\% & $\boldsymbol{\beta}$ & IC95\% & $\boldsymbol{\beta}$ & IC95\% & $\boldsymbol{\beta}$ & IC95\% \\
\hline IMC $^{\star}$ Apoio social do pai ${ }^{\star}$ & $-9,64$ & $-36,60 ; 17,32$ & 0,07 & $-4,32 ; 4,46$ & $-0,16$ & $-1,88 ; 1,57$ & $-0,09$ & $-5,63 ; 5,46$ \\
\hline $\mathrm{IMC}^{\star}$ Apoio social da mãe $\mathrm{e}^{\star \star}$ & $-16,89$ & $-46,75 ; 12,96$ & $-0,32$ & $-5,20 ; 4,55$ & $-0,29$ & $-2,20 ; 1,63$ & $-0,60$ & $-6,75 ; 5,54$ \\
\hline $\mathrm{IMC}^{\star}$ Apoio social do amigo ${ }^{\star * \star}$ & $-2,35$ & $-30,26 ; 25,57$ & 1,84 & $-2,70 ; 6,37$ & 0,35 & $-1,44 ; 2,14$ & 2,19 & $-3,55 ; 7,92$ \\
\hline $\mathrm{IMC}^{\star}$ Autoeficácia ${ }^{\star * * *}$ & $-43,35$ & $-148,30 ; 61,61$ & 7,81 & $-9,26 ; 24,89$ & 1,63 & $-5,10 ; 8,35$ & 9,44 & $-12,14 ; 31,01$ \\
\hline
\end{tabular}

Fonte: Elaborada pelos autores.

vidade física, como, por exemplo, locais e equipamentos para a prática, segurança ${ }^{27}$. Desse modo, os pais tenderiam a fornecer menos apoio para a prática de atividade física em função dessas condições ambientais mais adversas.

Outro fator a ser considerado é que as atividades físicas em que os adolescentes de baixo nível socioeconômico estão mais envolvidos não dependem de locais, equipamentos e incentivos específicos para a sua prática ${ }^{26}$. No presente estudo, a medida de atividade física considerou as atividades praticadas no lazer, na escola, nas aulas de educação de física e como forma de deslocamento. É possível que a maior parte do tempo de prática desses adolescentes seja em atividades físicas que não necessitem de apoio social dos pais: atividades nas aulas de educação física, deslocamento a pé ou de bicicleta para ir à escola e retornar para casa, brincadeiras e jogos ao ar livre.

No presente estudo, a autoeficácia não se associou à atividade física, contrariando os resultados de estudos prévios com adolescentes ${ }^{2,3}$. É possível que isso tenha ocorrido devido à escala utilizada para mensurar a autoeficácia, que, apesar de ter níveis satisfatórios de reprodutibilidade, alcançou baixos níveis de consistência interna e validade. As barreiras para a prática de atividade física que foram utilizadas para compor os itens da escala podem não ser representativas dos adolescentes que fizeram parte deste estudo. Desse modo, os níveis de autoeficácia não refletiriam as percepções dos adolescentes quanto a sua capacidade de superar barreiras para serem fisicamente ativos. Outra explicação é que as atividades físicas mais praticadas por eles podem não exigir a superação de barreiras que são identificadas para outras atividades físicas como, por exemplo, atividades físicas no lazer, esportes estruturados e exercícios físicos em academias ${ }^{28}$.

O estado nutricional dos adolescentes não moderou a associação do apoio social e da autoeficácia com o tempo de atividade física, sugerindo que as possíveis influências desses construtos sobre a atividade física têm o mesmo sentido e magnitude em adolescentes com e sem excesso de peso corporal, e independentemente do sexo dos adolescentes.

Ao contrário do que foi observado no presente estudo, a autoeficácia tem se mostrado associada a maior tempo de prática de atividade física de intensidade moderada-vigorosa em adolescentes com e sem excesso de peso, independentemente 
da medida de atividade física ${ }^{5,6,8}$. As mesmas explicações que foram apresentadas para a ausência de associação entre o apoio social, a autoeficácia e atividade física podem ser aplicadas para esses resultados: perfil socioeconômico, as atividades físicas mais praticadas pelos adolescentes do presente estudo e a escala de autoeficácia utilizada. Desse modo, a autoeficácia não se mostrou um preditor da atividade física, e sem diferenças em termos de possíveis influências na prática de atividade física dos adolescentes com e sem excesso de peso corporal.

Os resultados disponíveis até o momento indicam que adolescentes que recebem mais apoio social dos amigos apresentam maior prática de atividade física, independentemente do seu estado nutricional ${ }^{1,2}$, corroborando os resultados do presente estudo. Este é um achado importante para a promoção da prática de atividade física, tendo em vista que o apoio social dos amigos é um dos principais preditores dessa prática ${ }^{1}$, e a atividade física tem importante papel na prevenção, no controle e tratamento do excesso de peso e da obesidade ${ }^{2}$. Desse modo, favorecer o apoio social dos amigos é uma das formas de aumentar o nível de atividade física dos adolescentes de diferentes estados nutricionais. Além de promover vários benefícios para a saúde, isso pode ajudar na redução e no controle do excesso de peso e/ ou obesidade, bem como dos problemas de saúde associados a essa condição.

Mais estudos precisam ser conduzidos para avaliar o possível papel moderador do estado nutricional na influência do apoio social e da autoeficácia na prática de atividade física dos adolescentes. Esses estudos precisam considerar diferentes tipos de prática de atividade física, de fontes e tipos de apoio social, e as escalas de autoeficácia devem conter barreiras para prática de atividade física representativas da faixa etária e das atividades físicas mensuradas, envolvendo adolescentes de diferentes condições socioeconômicas.

Dentre as limitações do presente estudo, destacam-se estas: presença de um possível viés de seleção, devido à maior proporção de adolescentes de 12 a 14 anos de idade que não cumpriram os critérios de redução dos dados do acelerômetro, comparativamente aos de 10 a 11 anos. No entanto, não foram observadas diferenças significativas nas variáveis apoio social, autoeficácia e nível de atividade física entre os incluídos e excluídos nas análises. O fato de não se ter incluído escolares da rede privada pode ter contribuído para maior homogeneidade nos resultados da condição socioeconômica, prática de atividade física, apoio social e autoeficácia dos adolescentes. A escala de autoeficácia apresentou baixos níveis de consistência interna e de validade, o que pode ter colaborado para a ausência de associações significativas. Esses fatores podem ter contribuído para a ausência de associação entre o apoio social dos pais e a autoeficácia com a prática de atividade física dos adolescentes; bem como para a ausência de interação entre autoeficácia e apoio social com o estado nutricional dos adolescentes.

Dentre os pontos fortes deste estudo, destacam-se os seguintes: uso de amostra representativa e com poder estatístico adequado (1-beta igual ou superior a $80 \%$ ) para testar as hipóteses; utilização de instrumentos previamente validados, aplicados por equipe treinada e que não conhecia as hipóteses do estudo; a atividade física foi mensurada por meio de acelerômetro, evitando viés de memória e de mensuração, tendo em vista que adolescentes com excesso de peso tendem a superestimar o tempo de atividade física comparativamente aos de peso normal ${ }^{29}$.

Adolescentes que recebiam mais apoio social dos amigos apresentaram maior tempo de prática de atividade física, independentemente do seu estado nutricional (com ou sem excesso de peso corporal). No entanto, o apoio social dos pais e a autoeficácia não se associaram ao tempo de prática de atividade física. Os achados do presente estudo sugerem que intervenções para aumentar a atividade física com foco no apoio social dos amigos devem incluir ações similares para adolescentes com diferentes estados nutricionais. 


\section{Colaboradores}

TG Cabral, participou da concepção do manuscrito, coleta, análise e interpretação dos dados, e da redação do manuscrito. IRD Moura, participou da coleta, análise dos dados e redação do manuscrito. JC Farias Júnior, participou da redação e revisão crítica do manuscrito.

\section{Referências}

1. Bauman AE, Reis RS, Sallis JF, Wells JC, Loos RJ, Martin BW, Lancet Physical Activity Series Working Group. Correlates of physical activity: why are some people physically active and others not? Lancet 2012; 380(9838):258-271.

2. Rhodes RE, Janssen I, Bredin SS, Warburton DE, Bauman A. Physical activity: Health impact, prevalence, correlates and interventions. Health Psychol 2017; 32(8):942-975.

3. Cheng L, Mendonça G, Farias Júnior J. A associação entre fatores psicossociais e atividade física em adolescentes é moderada por fatores sociodemográficos? Rev Bras Ativ Fis Saude 2016; 21(4):297-306.

4. Trost SG, Kerr L, Ward DS, Pate RR. Physical activity and determinants of physical activity in obese and non-obese children. Int J Obes 2001; 25(6):822.

5. Kitzman-Ulrich $\mathrm{H}$, Wilson DK, Van Horn $\mathrm{ML}$, Lawman HG. Relationship of body mass index and psychosocial factors on physical activity in underserved adolescent boys and girls. Health Psychol 2010; 29(5):506.

\section{Financiamento}

Conselho Nacional de Desenvolvimento Científico e Tecnológico (CNPq) e Fundação de Apoio à Pesquisa do Estado da Paraíba (FAPESQ).
6. Bourdeaudhuij I, Lefevre J, Deforche B, Wijndaele K, Matton L, Philippaerts R. Physical ctivity and psychosocial correlates in normal weight and overweight 11 to 19 year olds. Obes Res 2005; 13(6):1097-1105.

7. George SM, Wilson DK, Lawman HG, Van Horn ML. Weight status as a moderator of the relationship between motivation, emotional social support, and physical activity in underserved adolescents. J Pediatr Psychol 2013; 38(4):387-397.

8. DS, Dowda M, Trost SG, Felton GM, Dishman RK, Pate RR. Physical activity correlates in adolescent girls who differ by weight status. Obesity 2006; 14(1):97105.

9. Mendonça G, Farias Júnior JC. Physical activity and social support in adolescents: analysis of different types and sources of social support. J Sports Sci 2015; 33(18):1942-1951.

10. Bandura A. Guide for constructing self-efficacy scales. Self-Efficacy Beliefs Adolesc 2006; 5(1):307-337. 
11. Ng M, Fleming T, Robinson M, Thomson B, Graetz N, Margono C, Mullany EC, Biryukov S, Abbafati C, Abera SF, Abraham JP, Abu-Rmeileh NME, Achoki T, AlBuhairan FS, Alemu ZA, Alfonso R, Ali MK, Ali R, Guzman NA, Ammar W, Anwari P, Banerjee A, Barquera S, Basu S, Bennett DA, Bhutta Z, Blore J, Cabral N, Nonato IC, Chang J-C, Chowdhury R, Courville KJ, Criqui MH, Cundiff DK, Dabhadkar KC, Dandona L, Davis A, Dayama A, Dharmaratne SD, Ding EL, Durrani AM, Esteghamati A, Farzadfar F, Fay DFJ, Feigin VL, Flaxman A, Forouzanfar MH, Goto A, Green MA, Gupta R, Hafezi-Nejad N, Hankey GJ, Harewood HC, Havmoeller R, Hay S, Hernandez L, Husseini A, Idrisov BT, Ikeda N, Islami F, Jahangir E, Jassal SK, Jee SH, Jeffreys M, Jonas JB, Kabagambe EK, Khalifa SEAH, Kengne AP, Khader YS, Khang Y-H, Kim D, Kimokoti RW, Kinge JM, Kokubo Y, Kosen S, Kwan G, Lai T, Leinsalu M, Li Y, Liang X, Liu S, Logroscino G, Lotufo PA, Lu Y, Ma J, Mainoo NK, Mensah GA 66, Merriman TR, Mokdad AH, Moschandreas J, Naghavi M, Naheed A, Nand D, Narayan KMV, Nelson EL, Neuhouser ML, Nisar MI, Ohkubo T, Oti SO, Pedroza A, Prabhakaran D, Roy N, Sampson U, Seo H, Sepanlou SG, Shibuya K, Shiri R, Shiue I, Singh GM, Singh JA, Skirbekk V, Stapelberg NJC, Sturua L, Sykes BL, Tobias M, Tran BX, Trasande L, Toyoshima H, van de Vijver S, Vasankari TJ, Veerman JL, Velasquez-Melendez G, Vlassov VV, Vollset SE, Vos T, Wang C, Wang XR, Weiderpass E, Werdecker A, Wright JL, Yang YC, Yatsuya H, Yoon J, Yoon S-J, Zhao Y, Zhou M, Zhu S, Lopez AD, Murray CJL, Gakidou E. Global, regional, and national prevalence of overweight and obesity in children and adults during 1980-2013: a systematic analysis for the Global Burden of Disease Study 2013. Lancet 2014; 384(9945):766-781.

12. Instituto Brasileiro de Geografia e Estatística (IBGE). Pesquisa Nacional de Saúde do Escolar (PeNSE) [Internet]. 2016 [acessado 2020 abr 4]. Disponível em: https://biblioteca.ibge.gov.br/visualizacao/livros/ liv97870.pdf.

13. Curtis P. The experiences of young people with obesity in secondary school: some implications for the healthy school agenda. Heal Soc Care Community 2008; 16(4):410-418.

14. Stankov I, Olds T, Cargo M. Overweight and obese adolescents: what turns them off physical activity?. Int J Behav Nutr Phys Act 2012; 9(1):1-15.

15. Fernández I, Canet O, Giné-Garriga M. Assessment of physical activity levels, fitness and perceived barriers to physical activity practice in adolescents: cross-sectional study. Eur J Pediatr 2017; 176(1): 57-65.

16. Barbosa AO, Prazeres Filho A, Farias Júnior JC. Effect of number of hours and days of accelerometer use on physical activity estimates in adolescents. Rev Bras Cineantropom Hum 2019; 21:55973.

17. Farias Júnior JC, Lopes ADS, Mota J, Hallal PC. Prática de atividade física e fatores associados em adolescentes no Nordeste do Brasil. Rev Saude Publica 2012; 46(3):505-515.

18. Associação Brasileira de Empresas de Pesquisa (ABEP). Critério de classificação econômica do Brasil [Internet]. 2014 [acessado 2015 nov 26]. Disponível em: http://wwwabeporg/new/codigosCondutasaspx.
19. Farias Júnior JC, Lopes ADS, Reis RS, Nascimento JVD, Borgatto AF, Hallal PC. Development and validation of a questionnaire measuring factors associated with physical activity in adolescents. Rev Bras Saude Mater Infant 2011; 11(3):301-312.

20. World Health Organization (WHO). WHO child growth standards: length/height-for-age, weight-for-age, weight-for-length, weight-for-height and body mass index-for-age: methods and development. Geneva: WHO; 2006.

21. Evenson KR, Catellier DJ, Gill K, Ondrak KS, McMurray RG. Calibration of two objective measures of physical activity for children. J Sports Sci 2008; 26(14):1557-1565.

22. Kirby J, Levin KA, Inchley J. Parental and peer influences on physical activity among Scottish adolescents: a longitudinal study. J Phys Act Health 2011; 8(6):785-793.

23. Wold B, Hendry L. Social and environmental factors associated with physical activity in young people. Young Active 1998; 119-132.

24. Fitzgerald A, Fitzgerald N, Aherne C. Do peers matter? A review of peer and/or friends' influence on physical activity among American adolescents. J Adolesc 2012; 35(4):941-958.

25. Kantomaa MT, Tammelin TH, Näyhä S, Taanila AM. Adolescents' physical activity in relation to family income and parents' education. Prev Med 2007; 44(5):410-415.

26. Stalsberg R, Pedersen AV. Effects of socioeconomic status on the physical activity in adolescents: a systematic review of the evidence. Scand J Med Sci Sports 2010; 20(3):368-383.

27. Sterdt E, Liersch S, Walter U. Correlates of physical activity of children and adolescents: a systematic review of reviews. Health Educ J 2013; 73(1):72-89.

28. Mendonça G, Cheng LA, Farias Júnior JC. Padrões de prática de atividade física em adolescentes de um município da região Nordeste do Brasil. Cien Saude Colet 2018; 23(7):2443-2451.

29. McMurray RG, Ward DS, Elder JP, Lytle LA, Strikmiller PK, Baggett CD, Young DR. Do overweight girls overreport physical activity? Am J Health Behav 2008; 32(5):538-546.

Artigo apresentado em 23/11/2019

Aprovado em 29/07/2020

Versão final apresentada em 31/07/2020

Editores-chefes: Romeu Gomes, Antônio Augusto Moura da Silva 